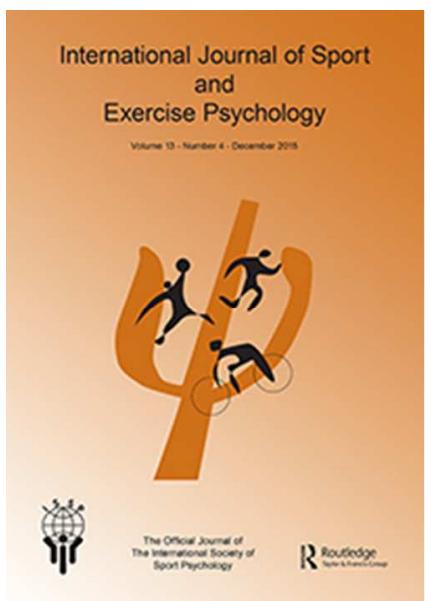

\title{
Investigating the Association of Self-Regulation of Learning Skills with Physical Activity in Hong Kong Chinese and Scottish adolescents
}

\begin{tabular}{|r|l|}
\hline Journal: & International Journal of Sport and Exercise Psychology \\
\hline Manuscript ID & RIJS-2016-0074 \\
\hline Manuscript Type: & Original Article \\
\hline Keywords: & self-regulation, physical activity, adolescents \\
\hline \multicolumn{2}{|l}{} \\
\end{tabular}

SCHOLARONE ${ }^{m}$

Manuscripts 
HONG KONG AND SCOTTISH ADOLESCENT SELF-REGULATION \& PHYSICAL ACTIVITY

Investigating the Association of Self-Regulation of Learning Skills with Physical Activity in Hong Kong Chinese and Scottish adolescents

\begin{abstract}
Purpose This study examines the role of self-regulated learning (SRL) skills in physical activity (PA) among Hong Kong Chinese (HK) and Scottish adolescents.

Methods Using a cross-sectional design, 480 HK (aged $11-19$; mean age $=14.93$ years, SD $=1.77$, male $=61.3 \%)$ and 411 Scottish, aged $12-16$ years $($ mean $=13.84 ;$ male $=47.9 \%)$ healthy adolescents participated in this study. PA was measured with the Godin LeisureTime Questionnaire (Godin \& Shephard, 1985). SRL was measured for Chinese (SRL-SRSC; Pitkethly \& Lau, 2015) and British adolescents (SLR-SRS; Toering et al., 2012). Results Moderate-to-vigorous intensity PA was significantly higher among Scottish $(M=$ 48.58; $S D=26.01 p=.0005)$ compared to HK adolescents $(M=31.14 ; S D=22.26)$. All SRL variables significantly explained a small amount of variance in moderate to vigorous intensity PA, in $\operatorname{HK}\left(\Delta R^{2}=.021, F(2,461)=6.320, p=.005\right)$ and $\operatorname{Scottish}\left(\Delta R^{2}=.020\right.$, $F(2,376)=8.427, p=.013)$ adolescents, reflection was a significant predictor (HK $p=.027$; $\mathrm{SC} p=.003)$
\end{abstract}

Conclusions In both cultures, higher self-regulating adolescents, may be more likely to engage in higher levels of PA. SRL has a significant impact on PA; and of all the SRL variables, reflection plays a key role in students engagement in PA.

\title{
Background
}

Globally, four out of five adolescents do not achieve recommended levels of physical activity (PA) (Hallal et al., 2012). Regular participation in PA is associated with a decreased risk of cardiovascular disease (Warburton et al. 2006), obesity and improvements in psychological functioning in adolescents (Biddle \& Asare, 2011). As activity levels track from adolescence to adulthood (Malina, 2001; Telama et al., 2005) young people are at risk 
HONG KONG AND SCOTTISH ADOLESCENT SELF-REGULATION \& PHYSICAL ACTIVITY

of developing into inactive adults along with the persistence of physical, psychological and social problems. Thus, improving physical activity levels is a public health priority. Physical activity levels are declining not only in wealthy countries, such as the United States (US) and the United Kingdom (UK), but also in low to middle-income countries, such as China. This decline in PA is considered an important contributor to the global obesity epidemic, as well as many non-communicable diseases (Ha, Abbott, Macdonald, \& Pang, 2009).

Hong Kong Chinese (HK) children are among the most inactive children in the world (Guldan, Cheung \& Chui, 1998; Adab \& Macfarlane, 1998). In 2012, only 12.8\% of adolescent boys and $4.2 \%$ of adolescent girls met the recommended levels of PA (Leisure and Cultural Services Department (LCSD), 2012). Additionally, the prevalence of obesity in HK for secondary school students, rose from $13.6 \%$ in $1997 / 1998$ to 18.7 in the $2010 / 2011$ school year (Department of Health, HK SAR, 2012). Within Europe, the UK is the third most inactive country and the eighth most inactive in the world (Hallal et al., 2012). Of the four nations that comprise the UK, Scotland and Northern Ireland have the highest prevalence of obesity, Scotland (68.3\%), Northern Ireland (69\%), England (67\%) and Wales (63\%) and, according to Scottish Government statistics, even though, $75 \%$ of $11-12$ year olds and 59\% of 13 - 15 year olds reported meeting the daily PA recommendations, $31.6 \%$ of children were overweight or obese and $15.7 \%$ children were obese or morbidly obese in 2011 (Gray \& Leyland, 2011). Both HK and Scotland's PA recommendations for 5-18 year olds are in line with the World Health Organisation (WHO, 2013), and both governments have set targets to improve activity levels. However, on an individual level, considerable effort and self-regulation (SR) is required to start and subsequently maintain an active lifestyle (Baumeister, Scmeichel \& Vohs, 2003).

Self-regulated Learning (SRL) is defined as "The extent to which individuals are metacognitively, motivationally and behaviourally proactive participants in their own learning and developmental processes" (Zimmerman 1986, p. 308). Bandura (2004) 
suggested that it is necessary to equip individuals with the relevant psychological skills and efficacy beliefs, to help them manage the daily multitude of emotional and social pressures, and to manage their environments and social relationships. SRL (Zimmerman, 1986) has an established role in the enhancement of development and learning in academic achievement (Zimmerman, Moylan, Hudesman, White \& Flugman, 2011), sport (Toering, ElferinkGemser, Jordet \& Visscher, 2009) and health settings (Kitsantas, 2000). It is not surprising to find that individuals with a tendency to take a more proactive approach to personal learning and development can also apply this to other areas. For example, talented athletes have been found to be high self-regulators and high academic achievers (Jonker, Elferink-Gemser, \& Visscher, 2009). Furthermore, metacognitive SRL skills such as planning, self-monitoring, self-evaluation, reflection as well motivational aspects of self-efficacy and effort are important, not only for performance improvements, as such an approach is capable of meeting the necessary theoretical demands for meaningful behaviour change research (Boekarts, 1997) and may be instrumental in enhancing levels of PA (Toering et al., 2012). In short, following Zimmerman's (1986) SRL model helps to explain how individuals are able to make improvements through a systematic method of learning how to adapt to ever changing environments (Schunk, 2001).

\section{SRL and Culture}

Research into SRL within academic settings has demonstrated cultural differences between Eastern and Western populations (Chen, Lee \& Stevenson, 1995; Purdie, Hattie \& Douglas, 1996; Stevenson \& Lee, 1996; Ho, 2004). In this field, Western scholars tend to describe Asian learners as being reactive rather than proactive in their approaches to learning, and more dependent on instruction rather than initiating their own learning (Turingan \& Yang, 2009). Purdie, Hattie \& Douglas, 1996 found that Australian students had a narrower school-based view of learning, and the Japanese students understood learning from a much broader perspective. Despite the differences in perspective, analysis of the 
HONG KONG AND SCOTTISH ADOLESCENT SELF-REGULATION \& PHYSICAL ACTIVITY

strategy use between the Japanese and Australian students was found to be similar. Pilay, Purdie and Boulton-Lewis (2000) also provided evidence against the commonly cited notion of passive Asian students (Kember, 2000), by assessing cross-cultural variations in Australian and Malay students using two well validated questionnaires in conceptions of learning (Purdie, Hattie \& Douglas, 1996); and motivated strategies for learning (Pintrich et al., 1991). This research identified a new factor termed 'learning as duty', which resulted in the only significant difference between the groups. The authors explained this finding through the collectivist nature of Malaysian society and the Asian phenomenon of 'loss of face'. This 'loss of face' might translate into 'duty to learn' and an obligation for the students to behave in a way expected by their families and communities. This finding supports the idea that Asian students, despite the collectivist culture notion, demonstrated 'self' responsibility and 'self' regulate their learning to benefit the collective ideal.

\section{SRL and PA}

With regards to SRL and adolescent PA, evidence suggests that interventions that manipulate SRL skills with the aim of improving PA are beneficial. For example, positive associations between SRL and PA have been found (Winters et al., 2003; Mathews \& Moran, 2011). With regards to experimental evidence, Lubans and Morgan (2008) designed a programme for secondary school students to promote lifestyle (e.g. walking/cycling to school) and lifetime physical activity. Goal setting and physical activity monitoring were the primary behaviour modification strategies focussed on and the results revealed significantly more steps being accumulated by adolescents who were inactive at baseline. Araújo-Soares et al. (2009) targeted variables from social cognitive theory, self-regulation theory and planning theory as evidenced mediators of PA change through a school-based intervention. Although no significant differences were found at post-test, at nine months the follow-up, the intervention group reported significantly more self-reported moderate-to-vigorous intensity 
PA. This suggests the long-term potential of impacting on PA behvaiours through variables such as those under the SRL umbrella.

Despite promising empirical support for the role of SR on PA among adolescents, research in this area is limited. For example, it is unclear which self-regulatory skills are most effective in facilitating PA behaviours, particularly among adolescents (Matthews \& Moran, 2011). Importantly, while a cross-sectional study, such as this may not explain SRL process, which would need to be investigated over time, as an initial step into this SRL-PA area, the current study sought to investigate which components of SRL, or SRL skills, might be linked to PA in both HK and Scottish adolescents, knowledge which may guide further PA enhancement research. Therefore, the current research aimed to answer the following questions: 1) How do levels of PA and SRL component scores compare between HK and Scottish adolescents? 2) Are self-regulatory components from a theoretically driven model associated with PA in HK and Scottish adolescents? 3) Do any differences exist in the contribution of SRL variables between moderate and vigorous intensity PA between HK and Scottish Adolescents?

\footnotetext{
Methods

Design and Participants

This study used a cross-sectional design, with convenience sampling of healthy adolescents with no physical or psychological contraindications to exercise. Recruitment was achieved by contacting physical education (PE) teachers via existing networks. Four hundred and eighty adolescents (age range $11-19 ;$ mean age $=14.9$ years, $\mathrm{SD}=1.8$, male $=294$ ) recruited from three secondary schools in HK participated in this study two secondary schools are government funded, English-medium (teaching language was English) secondary school, and the other is a government funded Chinese speaking school. All three schools represent a culturally representative sample. Four hundred and eleven Scottish adolescents, aged $12-16$ years $($ mean $=13.8 ; \mathrm{SD}=1.4 ;$ male $=197)$, from one culturally representative $-5-$
} 
HONG KONG AND SCOTTISH ADOLESCENT SELF-REGULATION \& PHYSICAL ACTIVITY

secondary school in Scotland, agreed to take part in this study. The Scottish school enrols students from unemployed to professional parents, from a wide catchment area covering four large towns on the west coast of Scotland.

\section{Measures}

SRL was assessed in the HK sample with the Chinese SRL self-report scale (SRLSRS-C; Pitkethly \& Lau, 2015; Cronbach' $\alpha$ coefficients, for all 6 subscales ranged from.72 to .89 ; ICC's range from .69 to .82 for all 6 subscales), which assesses dispositional SRL as a metacognitive and motivational construct (Toering et al., 2012). Self-reported SRL was measured in the Scottish sample with the SRL scale (SLR-SRS; Toering et al., 2012; $\alpha=.73$ to .85 for all 6 subscales; ICC's range from 0.70 to 0.84 for all 6 subscales).

For both the Chinese and English SRL measures total scores can be generated from all six components (planning, self-monitoring, effort, self-efficacy, self-evaluation, and reflection). Higher scores indicate a higher tendency for SRL. Component scores can be calculated separately for each of the six components and are interpreted in the same way as the total score. For example, higher planning scores indicate better planning capabilities. An example of an item from the planning scale is 'I carefully plan my course of action to solve a problem'.

Self-Reported PA In this study leisure-time PA was examined as it is considered one of the most important dimensions of PA (Tudor-Locke, Ainsworth \& Popkin, 2001) and can positively impact on the uptake of lifelong exercise (Sallis \& Owen, 1999). The Godin Leisure-Time Exercise Questionnaire (GLTEQ) was used to measure leisure-time PA (Godin \& Shephard, 1985) in both samples. This measure has been validated in a number of studies with adolescents (Hortz \& Petosa, 2008; Matthews \& Moran, 2011), test-retest reliability of .81 (Sallis, Buono, Roby, Micale, \& Nelson 1993).

\section{Procedure}


HONG KONG AND SCOTTISH ADOLESCENT SELF-REGULATION \& PHYSICAL ACTIVITY

Ethical approval was obtained and after the PE teachers had declared their interest to take part in the study, upon receipt of written consent from the principals, all parents were contacted and thereafter provided written informed consent. All participants assented prior to questionnaire completion. Questionnaires were administered during school hours as determined by class teachers. Researchers and research assistants were available at data collection times to clarify any issues.

\section{Analyses}

SPSS 21.0 (IBM Corp., 2012; version 21.0) was used for all data analyses. SRL and PA data that were missing were dealt with by listwise exclusion. As the recommended daily guidelines (WHO, 2013) for adolescent PA are 60 minutes of moderate-to-vigorous PA, the GLTEQ data analysis focused on moderate and vigorous scores and excluded the light activity scores (Matthews \& Moran, 2011). All data were assessed for outliers and normality to ensure the data were fit for statistical analyses.

Pearson product correlations were conducted to analyse the relationship between gender and PA, and among SRL variables and PA. Independent t-tests were conducted in both samples to examine PA, SRL and gender mean differences. Measurement invariance was conducted to confirm the equality of factor structures across the HK and Scottish groups using AMOS (within SPSS 21.0; IBM Corp., 2012; version 21.0). According to crosscultural literature, in order to meaningfully compare differences between cultures, measurement invariance should be conducted. In addition to measure adaptation and validation (SRL-SRS; Toering et al., 2012; and SRL-SRS-C; Pitkethly \& Lau, 2015), a further three levels of invariance (configural invariance, metric invariance and scalar invariance) require to be fulfilled (e.g. Horn \& McArdle, 1992; Byrne, 2004). Within a multi-group CFA (MGCFA; Milfont \& Fischer, 2010), configural invariance was tested by cross-validating the factor structure across groups. A covariance matrix was analysed and a maximum likelihood method of estimation was used. In this model one factor loading was 
HONG KONG AND SCOTTISH ADOLESCENT SELF-REGULATION \& PHYSICAL ACTIVITY

set to be equal in each group. The metric invariance model set all factors loading to be equal across groups. The scalar invariance model (model 3) set all factor loadings, item intercepts and residual variances equal across groups. A structural invariance model (model 4) set all factor loadings, item intercept, residual variances and factor variances equal across groups. Additionally, a model with equal variances was examined.

Several fit indices were used to determine measurement invariance across groups. Model fit was assessed with $\chi^{2}$; comparative fit index (CFI; Bentler, 1990); Tucker-Lewis index (TLI); Bentler \& Bonett, 1980) criterion > .90 (Hu \& Bentler, 1999), and the root square mean error of approximation (RMSEA; Steiger, 1990), MacCallum, Browne and Sugawara (1996) have suggested that $0.01,0.05$, and 0.08 to indicate excellent, good, and mediocre fit, respectively. The RMSEA 95\% confidence intervals are also presented. Additionally, the change in CFI ( $\triangle \mathrm{CFI})$ was assessed and values of above .01 indicate decreasing fit (Cheung \& Rensvold, 2002). Full measurement invariance of a given type (e.g., metric, scalar) is frequently not satisfied in practice and a minimal degree of partial measurement invariance is necessary for comparisons of cross-national differences in factor means to be meaningful (Steenkamp and Baumgartner 1998). Subsequently, after confirming the measurement invariance across both samples of students, independent t-tests were conducted to test differences between SRL components.

Assumptions of hierarchical multiple regression (HMR) were tested and found to be acceptable in both samples including, normality, and independence of residuals, linear relationships, homoscedasticity, and collinearity statistics. The range of ages in both samples was taken into consideration by including age in the first block of the HMR analyses along with gender. Thereafter, the six SRL variables were entered into HMR analyses according to social cognitive theory (SCT). Self-reported PA (separate analysis for moderate, vigorous or moderate-to-vigorous PA) was the dependent variable; gender and age, followed by self- 
HONG KONG AND SCOTTISH ADOLESCENT SELF-REGULATION \& PHYSICAL ACTIVITY

efficacy, and subsequently the remaining SRL variables were entered as predictors in steps, according to their hierarchy in Zimmerman's model (see Zimmerman, 2000).

\section{Results}

Means, standard deviations and Pearson correlations for moderate, vigorous and both moderate-to-vigorous intensity PA and self-regulation variables in the HK and Scottish samples (see table 1).

\section{***Insert Table 1 here}

\section{PA Differences between HK and Scotland}

There was a statistically significant difference in moderate intensity PA between the HK $(M=10.93 ; S D=10.88)$ and Scottish adolescents, $(M=17.54 ; S D=12.36$; mean difference $=-6.9 ; 95 \%$ CI $[-8.51,-5.39] t(780)=-8.73 ; p=.0005)$. A statistically significant difference was also found for vigorous intensity in the Scottish $(M=31.04 ; S D=20.04)$ adolescents compared to HK $(M=20.22 ; S D=16.45$, mean difference $=-11.3 ; 95 \% \mathrm{CI}[-$ $13.83,-8.81] ; t(746)=-8.85 ; p=.0005)$. Moderate-to-vigorous intensity PA was also significantly higher among Scottish adolescents $(M=48.58 ; S D=26.01$; mean difference = $18.3 ; 95 \%$ CI $[-21.52,-14.98], t(773)=-10.96 ; p=.0005)$ compared to HK $(M=31.14 ; S D=$ 22.26). Eleven per cent of HK adolescents reported engaged in moderate activity every day of the week and $4 \%$ in vigorous intensity PA. While $23.4 \%$ of Scottish adolescents reported engaging in moderate and $17 \%$ in vigorous intensity PA on seven days a week.

\section{SRL and PA}

A weak but significant relationship was found between total SRL scores and moderateto-vigorous PA for both HK $(n=475 ; r=.17, p=.0001)$ and Scottish adolescents $(n=391 ; r$ $=.17, p=001)$. Pearson correlations between SRL variables and self-reported PA for both samples are presented in table 2.

$* * *$ Insert Table 2 here

$$
-9-
$$


HONG KONG AND SCOTTISH ADOLESCENT SELF-REGULATION \& PHYSICAL ACTIVITY

\section{Measurement Invariance of SRL}

Initially, a configural model fitted the data adequately, followed by a metric model, constraining corresponding factor loadings to be equal across the HK and the Scottish group, which fitted the data adequately and showed that the $\triangle \mathrm{CFI}$ value between the configural and the metric model was less than the criterion of .01 ( $\triangle \mathrm{CFI}=.001$; Cheung \& Rensvold, 2002). Scalar invariance was then tested which imposed constraints on the item corresponding item intercepts but this model did not meet the $\triangle \mathrm{CFI}$ criterion. As a result, a partial scalar model (see table 3) freely estimated these parameters in both groups and provided evidence of partial scalar invariance $(\triangle \mathrm{CFI}=.006)$. The subsequent model tested partial error invariance, this model was accepted using the $\triangle \mathrm{CFI}$ criterion $(\triangle \mathrm{CFI}=.004)$. Overall, evidence of partial measurement invariance analysis was provided which indicate that the factorial structure, the slopes and intercepts, and variances of the SRL construct are similar between the HK and the Scottish samples. Table 3 presents the measurement invariance results.

\section{***Insert Table 3 here}

\section{SRL Component Differences between HK and Scotland}

Total mean SRL score was significantly higher for Scottish adolescents $(M=132.93$; $S D=21.41 ; 95 \%$ CI $[-47.84,-43.09], t(619)=-37.65, p=.0005)$ compared to HK adolescents $(M=86.93 ; S D=12.49)$.

For Scottish adolescents, self-monitoring $(M=2.61 ; S D=.56 ; 95 \%$ CI [-.23,-..07]; $t(888)=-3.849 ; p=.0005)$, self-evaluation $(M=3.51 ; S D=.73 ; 95 \%$ CI $[-.28,-.09]$; $t(796.034)=-4.070 ; p=.0005)$ and reflection $(M=3.80 ; \mathrm{SD}=.59 ; 95 \%$ CI $[-.23,-.06]$; $t(858.247)=-3.506 ; p=.0005)$ were significantly higher compared to HK adolescents. Planning $(p=.931)$, effort $(p=.116)$ and self-efficacy $(\mathrm{p}=.056)$ were not significantly different between HK and Scottish adolescents. 
HONG KONG AND SCOTTISH ADOLESCENT SELF-REGULATION \& PHYSICAL ACTIVITY

\section{Hierarchical Multiple Regression (HMR)}

HK. Only the model containing age, gender and self-efficacy significantly predicted moderate intensity PA $\left(\Delta R^{2}=.020, F(1,466)=3.996, p=.002\right)$. None of the other SRL variables significantly contributed to the prediction of moderate intensity PA. For vigorous intensity PA, controlling for age and gender, the final model containing all self-regulatory variables was significant $\Delta R^{2}=.018, F(2,461)=6.620, p=009$; adjusted $R^{2}=.035$. The SRL variables explained $4.7 \%\left(R^{2}\right.$ model 5 minus $R^{2}$ model $\left.1=.047\right)$ i.e. all SRL predictors minus gender and age $\left.\left(R^{2}=.047\right)\right)$ of the variance of vigorous intensity PA. Of the SRL variables, self-evaluation $(p=.03)$ significantly predicted PA. For moderate-to-vigorous intensity PA, $5.9 \%\left(R^{2}=.059\right)$ of the variance was explained by the model containing all SRL variables $\left(\Delta R^{2}=.021, F(2,461)=6.320, p=.005\right.$; adjusted $R^{2}=.048$, with reflection $(p$ $=.027$ ) being the only significant predictor.

Scotland Only the model containing age, gender and self-efficacy predicted $\left(\Delta R^{2}\right.$ $=.010, F(1,381)=1.328, p=.048$; adjusted $\left.R^{2}=.003\right)$ moderate intensity PA. For vigorous intensity PA, the final model containing all SRL variables $\left(R^{2}\right.$ model 5 minus $R^{2}$ model $1=$ $.062)$ predicted $6.2 \%$ of the variance in vigorous intensity $\mathrm{PA}\left(\Delta R^{2}=.016, F(2,376)=\right.$ $11.422, p=.027$; adjusted $\left.R^{2}=.178\right)$. In this model self-efficacy $(p=.008)$ and reflection $(p$ $=.008)$ were significant SRL predictors. All SRL variables $\left(R^{2}\right.$ model 5 minus $R^{2}$ model $1=$ $.068)$ significantly explained $6.8 \%$ of the variance in moderate-to-vigorous intensity $\mathrm{PA}\left(\Delta R^{2}\right.$ $=.020, F(2,376)=8.427, p=.013$; adjusted $\left.R^{2}=.134\right)$, with self-efficacy $(p=.003)$ and reflection $(p=.003)$ as the significant predictors.

$* * *$ Insert Table 4 here 
SELF-REGULATED LEARNING \& ADOLESCENT PHYSICAL ACTIVITY

\section{Discussion}

The current study found that Scottish students engaged in significantly more PA than HK students, which was associated with higher self-monitoring, self-evaluation and reflection scores. This study has also demonstrated a similar pattern between SRL and PA in both adolescent groups with reflection playing a key role in predicting moderate-to-vigorous intensity PA in both samples.

\section{How do levels of PA and SRL component scores compare between HK and Scottish} adolescents?

Despite the fact that the Scottish adolescents engaged in significantly more PA than HK adolescents, neither adolescent group engaged in the recommended amount of PA. These results highlight the urgency for PA promotion strategies for $\mathrm{HK}$ adolescents in particular. In addition to the fact that Scottish adolescents engaged in more PA than Chinese adolescents, they also used more self-monitoring, self-evaluation, and reflection. Self-monitoring has been identified as one of the most important behaviour change techniques to have a positive effect on PA (Olander et al., 2013). The Scottish adolescents in the current study may have benefited from the fact that self-monitoring provides important information for setting appropriate goals and for the evaluation of progress towards those goals and affects personal competence and self-esteem.

The Scottish adolescents' higher self-evaluation scores represent a higher ability to assess behaviours for accuracy and error towards the execution of a set plan (Toering et al., 2009). Educational psychology research has demonstrated that providing frequent selfevaluation opportunities strengthen self-efficacy and raise achievement outcomes for children and college students (Schunk \& Swartz, 1993, 1993b; Schunk \& Ertmer, 1999). The strengthening of self-efficacy, in turn, operates on behaviours through the mediating effects 
SELF-REGULATED LEARNING \& ADOLESCENT PHYSICAL ACTIVITY

of planning, self-monitoring, self-evaluation and reflection (Bandura, 1989; Zimmerman, 2002).

The Scottish adolescents appear to also have higher reflective abilities. The importance of reflection in learning, stressed by Ertmer and Newby (1996), enables thoughts to be translated into action. In this way a learner, or a developer, is able to comprehend newly acquired knowledge and skills, and apply these to a variety of situations (Peltier, Hay \& Drago, 2006). It has long been understood that we learn more from reflection than actual experiences (Dewey, 1933). This ability is crucial considering the dynamic interactions between personal, environmental and behavioural factors which adolescents face in daily life. Although limited research is available to compare the current study's links between reflection and adolescents PA, elite level sport performance among adolescents shows reflection to be the most important factor related to superior performance, and distinguishes the best performers (Cleary \& Zimmerman, 2001; Toering et al., 2009; Jonker et al., 2012).

\section{Are self-regulatory components from a theoretically driven model associated with PA in HK and Scottish adolescents?}

With regards to the contribution of SRL to adolescent PA, the current study has added weight to current knowledge by assessing six SRL variables from Zimmerman's model, and revealing that these variables weakly but significantly predicted $6.1 \%$ of the variance in HK adolescents and $7.5 \%$ of the variance Scottish adolescents for moderate-to-vigorous intensity PA. The current findings are comparable to Winters et al. (2003) who found that selfregulation accounted for $8 \%$ of variance in vigorous PA and $6 \%$ of the variance in moderate PA. Mathews and Moran (2011), found that three SRL variables explained 10.7\% in moderate-to-vigorous intensity PA.

Although current findings only demonstrate a small amount of significant explained variance, the amount is comparable with other studies and is considered important. It is 
widely acknowledged in social-cognitive theory and PA related literature, that most of the PA variance remains unexplained and that further research is needed (Plotnikoff, Costigan, Karunamuni \& Lubans, 2013). According to Rosenthal (1990) small amounts of variance can result in big practical differences. Indeed, Epstein (1979) suggested that low predictive power is expected when attempting to predict a single behaviour from a single personality trait, however, when aggregating behaviour across occasions, the predictability of behaviour from personality traits increases. The fact that individual human behaviour has low reliability is a well understood phenomenon in psychometrics (e.g. Spearman-Brown prophecy formula).

In terms of the cultural differences, the current results show that the contribution of SRL variables to PA is similar in both cultures as all SRL variables explained a similar amount of variance in PA $(5.9 \%$ for the HK and $6.8 \%$ for the Scottish adolescents; see table 4). In addition, reflection played the most important in the SRL-PA relationship role in both samples. This may indicate that, despite the collectivist and individualist differences between the two cultures (Triandis, 1995) and the commonly cited notion of passive Asian students (Turingan \& Yang, 2009), it appears for this study that, whether SRL strategies are used for the individual or for the collective benefit, SRL variables contribute similarly towards PA in both cultures as they do in academic SRL research (Purdie et al., 1996; Pilay et al. 2000).

A cross-sectional study with HK adolescents conducted by Ho (2004) concluded that although SRL was related to academic achievement, mean SRL scores indicated much less frequent use of strategies compared to students from other countries on the same measure. Ho (2004) suggested that perhaps other cultural factors may have a powerful influence on achievement for HK students, such as parental expectations (Chen, Lee \& Stevenson, 1995) and cultural values in education (Ho, 1994). In a similar vein, the current study found that SRL is related to PA in HK adolescents, although the HK asolescents indicated much less use of self-monitoring, self-evaluation and reflection, which was associated with lower PA levels. 
SELF-REGULATED LEARNING \& ADOLESCENT PHYSICAL ACTIVITY

Thus, other cultural factors may be at play, for example, Yu, Chan, Cheng, Sung and Hau (2006) point out academic success is widely regarded as the only indicator of success in Chinese culture, and too much PA is generally discouraged as it is believed to drain energy and affect concentration.

Although the current study may not explain a large amount of the variation in PA from SRL, it is a lack of SR that contributes to problem behaviours such as overeating and inactivity. Tangney, Baumeister, and Boone (2004) identified that individuals with better scores in self-control had better grades, better relationships, higher self-esteem, healthier emotional lives and fewer impulse control problems, including over-eating. Therefore, the important evidence provided by the current study of the link between SRL and PA suggests that further SRL-PA research is warranted.

\section{Do any differences exist in the contribution of SRL variables between moderate and vigorous intensity PA between HK and Scottish Adolescents?}

In terms of SRL variables, the current study found that only self-efficacy predicted moderate intensity PA for both HK and Scottish adolescents. Thus, it seems self-efficacy beliefs alone, without any other SRL variables, may be sufficient for adolescents to engage in moderate intensity PA. Self-efficacy is clearly important in the adoption of physical activity (Bandura, 1989), and it is a putative mediator of successful interventions to increase PA. Winters et al. (2003) also demonstrated behavioural differences between self-reported moderate and vigorous intensity PA. The authors found that SR significantly accounted for $6 \%$ of the variance in moderate intensity PA. The differences found were due to varied contributions of selected SCT variables to either moderate or vigorous intensity PA.

However, beliefs alone may be insufficient to overcome behavioural inertia due to the fact that beliefs are only passive evaluations of behaviour (Baumeister, Heatherton \& Tice, 1994). The current study shows that a combination of all the six measured SRL 
variables appear to contribute to higher intensity (vigorous) PA in both the HK and the Scottish adolescents. In the HK sample, all six SRL variables explained $4.7 \%$ of the variance in vigorous PA, and $6.2 \%$ in the Scottish sample. A similar level (8\%) of unique explained variance in vigorous PA was found by Winters et al. (2003).

The significant predictors for vigorous PA in the HK and the Scottish models varied. Self-evaluation predicted vigorous intensity PA in the HK sample, and both self-efficacy and reflection did so in the Scottish sample. These findings not only confirm important role of self-efficacy in engaging at any intensity of PA, but also highlights the importance of Zimmerman's self-reflective phase in PA, which includes self-evaluation and reflection. The linear relationship between physical activity and health status is well documented (e.g. Warburton, 2006), so for improved cardiovascular fitness, in healthy adolescents, high intensity and high volume activty is strongly encouraged ( $>80$ minutes per day; e.g. Eliakim Makowksi, Brasel \& Cooper, 2000). The value of self-efficacy beliefs for encouraging moderate intensity physical activity levels is important to consider, especially for unfit or inactive adolescents, until they are able to engage in gradually higher intensity, vigorous activities (Gutin, Yin, Humphries\& Barbeau, 2005). However, to encourage higher intensity vigorous activity it may be important to focus on the combination of and the cyclical nature of SRL variables with particular attention to the reflective phase.

\section{Limitations}

Limitations of the current study include the cross-sectional design which prohibit causal inferences. SRL has to be studied over time, and therefore a cross-sectional study cannot tell us anything about learning as there are no reference points to observe potential change. However, this study has investigated the use of important SRL skills that are associated with PA, across culture, and as a result acts as a basis for future work investigating these skills. Additionally, the use of the Godin PA questionnaire is limited as it gathers 
SELF-REGULATED LEARNING \& ADOLESCENT PHYSICAL ACTIVITY

limited PA information. Typical criticisms of PA self-report data apply in this study, such as, results are highly dependent on social desirability, respondent's cognition, the day of the week as well as the time of day, and the recall of the intensity of PA is a limitation. The cross-cultural aspect of this research is also limited in its scope, and requires further extensive investigation importantly with repeated measures to investigate underlying patterns of the SRL processes, and not only comparing other cultures, but other age groups.

\section{Future Research}

Explicitly training SRL skills, by specifically focussing on teaching adolescents what and how to plan and monitor, what to evaluate and reflect on and how to do so in a specific context is important. Thus, future research should devise interventions that teach SRL skills in a PA context to prompt real-life ecological behaviour which may help to prevent the adolescent drop in PA motivation at crucial development stages (Brodersen et al., 2006;) and contribute to improved continuing healthy behaviours into adulthood. Specifically, the predictive value of reflection may be useful in identifying adolescents with higher and/or lower reflective or self-regulative abilities and intervening accordingly. In terms of PA specifically, PE teachers could use the SRL questionnaire to with their students. The focus on the six SRL aspects would be a beneficial addition to the content of PE lessons to encourage lifelong PA behaviours. Importantly, as noted previously, longitudinal research is warranted before stronger conclusions can be made.

\section{Conclusions}

In the current study, a cross-sectional analysis of PA revealed that although Scottish adolescents engaged in higher levels, both Scottish and HK adolescents engaged in insufficient levels of PA. SRL variables including planning, self-monitoring, self-evaluation, self-efficacy, effort and reflection are significantly associated with PA in both countries, with reflection the most important factor in this relationship. Self-regulation development does not 
occur naturally, but rather only in inspiring environments in which goal-setting and appropriate feedback play a crucial role (Boekarts \& Corno, 2005). Therefore, it is suggested that a focus on providing opportunities for young people to develop SRL skills may be a fruitful method of encouraging engagement in health benefiting PA.

\section{References}

Adab, P., Macfarlane, D. (1998) Exercise and health: new imperatives for public health policy in Hong Kong. Hong Kong Medical Journal. 4, 389-393.

Araújo-Soares, V., McIntyre, T., MacLennan, G., \& Sniehotta, F. F. (2009). Development and exploratory cluster-randomised opportunistic trial of a theory-based intervention to enhance physical activity among adolescents. Psychology and Health, 24, 805822. doi:10.1080/08870440802040707

Bandura, A. (1989). Human agency in social cognitive theory. American Psychologist, 44, 1175-1184. doi:10.1037/0003-066X.44.9.1175

Bandura, A. (2004). Health Promotion by Social Cognitive Means. Health Education Behavior, 31, 143-164. doi: 10.1177/1090198104263660

Baumeister, R. F., Heatherton, T. F., \& Tice, D. M. (1994). Losing control: How and why people fail at self-regulation. Academic Press.

Baumeister, R. F., Scmeichel, B. J., \& Vohs, K. D. (2003). Self-regulation and the executive function of the self. In A. W. Kruglanski \& E.T. Higgins (Eds.), Social psychology: Handbook of basic principles (Second edition) (p. 197-217). New York: Guilford.

Bentler, P. M. (1990). Comparative fit indexes in structural models. Psychological Bulletin, 107, 238 - 246. doi: 10.1037/0033-2909.107.2.238 
SELF-REGULATED LEARNING \& ADOLESCENT PHYSICAL ACTIVITY

Bentler, P. M., \& Bonett, D. G. (1980). Significance tests and goodness of fit in the analysis of covariance structures. Psychological bulletin, 88, 588. doi: 10.1037/00332909.88.3.588

Biddle, S. J., \& Asare, M. (2011). Physical activity and mental health in children and adolescents: a review of reviews. British journal of sports medicine. doi:10.1136/bjsports-2011-090185

Boekaerts, M. (1997). Self-regulated learning: A new concept embraced by researchers, policy makers, educators, teachers, and students. Learning and instruction, 7, 161186. doi:10.1016/S0959-4752(96)00015-1

Boekaerts, M., \& Corno, L. (2005). Self-Regulation in the Classroom: A perspective on Assessment and Intervention. Applied Psychology: An International Review, 54(2) 199-231. doi: 10.1111/j.1464-0597.2005.00205.x

Brodersen, N. H., Steptoe, A., Boniface, D. R., \& Wardle, J. (2007). Trends in PA and sedentary behaviour in adolescence: ethnic and socioeconomic differences. British journal of sports medicine, 41, 140-144. doi:10.1136/bjsm.2006.031138

Byrne, B. M. (2004). Testing for multigroup invariance using AMOS graphics: A road less travelled. Structural Equation Modeling, 11(2), 272-300. doi:10.1207/s15328007sem1102_8

Chen, C., Lee, S. Y., \& Stevenson, H. W. (1995). Response style and cross-cultural comparisons of rating scales among East Asian and North American students. Psychological Science, 170-175. doi: 10.1111/j.1467-8624.1995.tb00932.x

Cheung, G. W., \& Rensvold, R. B. (2002). Evaluating goodness-of-fit indexes for testing measurement invariance. Structural equation modeling,9(2), 233-255. doi:10.1207/S15328007SEM0902_5 
SELF-REGULATED LEARNING \& ADOLESCENT PHYSICAL ACTIVITY

Cleary, T.J., \& Zimmerman, B.J. (2001). Self-regulation differences during athletic practice by experts, non-experts and novices. Journal of Applied Sport Psychology, 13, 185206. doi: $10.1002 /$ pits. 10177

Department of Health Hong Kong, SAR. (2010). Action Plan to Promote Healthy Diet and PA Participation in Hong Kong. Retrieved October 2012, from http://www.change4health.gov.hk/filemanager/common/image/strategic_framework/ action_plan/action_plan_e.pdf

Dewey, J. (1933). How we think. Buffalo, NY: Prometheus Books.

Eliakim, A, Makowski, G.S., Brase, J.A., \& Cooper, D.M. (2000). Adiposity, lipid levels, and brief endurance training in nonobese adolescent males. International Journal of Sports Medicine, 21, 332-7.

Epstein, S. (1979). The stability of behavior: I. on predicting most of the people most of the time. Journal of Personality and Social Psychology, 37, $1097\{1126$. doi:

$10.1037 / 0022-3514.37 .7 .1097$

Ertmer, P. A., \& Newby, T. J. (1996). The expert learner: Strategic, self-regulated, and reflective. Instructional science, 24(1), 1-24.

Godin, G. \& Shephard, R. J. (1985). A simple method to assess exercise behavior in the community. Canadian Journal of Sport Science. 10, 141-146. 10, 141-146. Retrieved October 2012, from: http://www.dapatoolkit.mrc.ac.uk/documents/en/God/Godin_Leisure-Time_Exercise_Q.pdf.

Gray, L., \& Leyland, A. H. The Scottish Health Survey: Volume 2 - Children (2011). Retrieved January 2013, from http://www.scotland.gov.uk/Publications/2012/09/3327/43 
SELF-REGULATED LEARNING \& ADOLESCENT PHYSICAL ACTIVITY

Guldan, G. S., Cheung, I. L. T., \& Chui, K. K. H. (1998). Childhood obesity in Hong Kong: embracing an unhealthy lifestyle before puberty. International Journal of Obesity, 22(Supplement 4), S16.

Gutin, B., Yin, Z., Humphries, M. C., \& Barbeau, P. (2005). Relations of moderate to vigorous physical activity to fitness and fatness in adolescents. American Journal of Clinical Nutrition, 81, 746-750.

Ha, A., Abbott, R., Macdonald, D. and Pang, B. (2009). Comparison of perceived support for physical activity and physical activity related practices of children and young adolescents in Hong Kong and Australia. European Physical Education Review, 15. 155 - 173. doi: $10.1177 / 1356336 X 09345219$

Hallal, P. C., Andersen, L. B., Bull, F. C., Guthold, R., Haskell, W., \& Ekelund, U. (2012). Global physical activity levels: surveillance progress, pitfalls, and prospects. Lancet, 380, 247-57. doi: 10.1016/S0140-6736(12)60646-1

Ho, E. S. C. (2004). Self-regulated learning and academic achievement of Hong Kong secondary school students. Education Journal, 32(2), 87-107.

Horn, J. L., \& McArdle, J. J. (1992). A practical and theoretical guide to measurement invariance in aging research. Experimental aging research, 18(3), 117-144. doi:10.1080/03610739208253916

Hortz, B., \& Petosa, R. (2008). Impact of the "Planning to be Active" leisure time physical exercise program on rural high school students. Journal of adolescent health, 39, 530-535. doi:10.1016/j.jadohealth.2006.03.015

Hu, L. T., \& Bentler, P. M. (1999). Cutoff criteria for fit indexes in covariance structure analysis: Conventional criteria versus new alternatives. Structural Equation Modeling: A Multidisciplinary Journal, 6, 1-55. doi:10.1080/10705519909540118 
SELF-REGULATED LEARNING \& ADOLESCENT PHYSICAL ACTIVITY

IBM Corp. Released 2012. IBM SPSS Statistics for Windows, Version 21.0. Armonk, NY: IBM Corp.

Jonker, L., Elferink-Gemser, M. T., de Roos, I. M., \& Visscher, C. (2012). The role of reflection in sport expertise. Sport Psychologist, 26(2), 224.

Kember, D. (2000). Misconceptions about the learning approaches, motivation and study practices of Asian students. Higher education, 40(1), 99-121.

Kitsantas, A. (2000). The role of self-regulation strategies and self-efficacy perceptions in successful weight loss maintenance. Psychology and Health, 15, 811-820. doi:10.1080/08870440008405583

Leisure and Cultural Services Department of Hong Kong. (LCSD). Health exercise for all campaign-physical fitness test for community: final summary report. (2012).Retrieved November 2014, from http://www.lcsd.gov.hk/healthy/physical_fitness/download/SummaryReport_en.pdf

Lubans, D., \& Morgan, P. (2008). Evaluation of an extra-curricular school sport programme promoting lifestyle and lifetime activity for adolescents. Journal of sports sciences, 26, 519-529. doi:10.1080/02640410701624549

MacCallum, R. C., Browne, M. W., \& Sugawara, H. M. (1996). Power analysis and determination of sample size for covariance structure modeling. Psychological methods, 1(2), 130. doi:10.1037/1082-989X.1.2.130

Malina, R. M. (2001). Physical activity and fitness: pathways from childhood to adulthood. American Journal of Human Biology, 13(2), 162-172. doi: 10.1002/1520$6300(200102 / 03)$

Matthews, J., \& Moran, A. (2011). PA and self-regulation strategy use in adolescents. American Journal of Health Behavior, 35, 807-14. doi: 10.5993/AJHB.35.6.16 
SELF-REGULATED LEARNING \& ADOLESCENT PHYSICAL ACTIVITY

Milfont, T. L., \& Fischer, R. (2010). Testing measurement invariance across groups: Applications in cross-cultural research. International Journal of psychological research, 3(1), 111-130.

Olander, E. K., Fletcher, H., Williams, S., Atkinson, L., Turner, A., \& French, D. P. (2013).

What are the most effective techniques in changing obese individuals' physical activity self-efficacy and behaviour: a systematic review and metaanalysis. International Journal of Behavioral Nutrition and Physical Activity, 10(29), $1-15$.

Peltier, J. W., Hay, A., \& Drago, W. (2006). Reflecting on reflection: scale extension and a comparison of undergraduate business students in the United States and the United Kingdom. Journal of Marketing Education, 28, 5-16. doi: $10.1177 / 0273475305279658$

Pillay, H., Purdie, N. O. L. A., \& Boulton-Lewis, G. (2000). Investigating cross-cultural variation in conceptions of learning and the use of self-regulated strategies. Education journal Hong Kong Chinese university of Hong Kong, 28(1), 65-84.

Pintrich, P. R., Smmith, D., Garcia, T., McKeachie, W. (1991). The motivated strategies for learning questionnaire (MSLQ). Ann Arbor, MI: NCRIPTAL, The University of Michigan.

Pitkethly, A. J., \& Lau, P. W. (2015). Reliability and validity of the short Hong Kong Chinese Self-Regulation of Learning Self-Report Scale (SRL-SRS-C).International Journal of Sport and Exercise Psychology, 1-17. doi:10.1080/1612197X.2015.1025810

Plotnikoff, R. C., Costigan, S. A., Karunamuni, N., \& Lubans, D. R. (2013). Social cognitive theories used to explain physical activity behavior in adolescents: a systematic 
SELF-REGULATED LEARNING \& ADOLESCENT PHYSICAL ACTIVITY

review and meta-analysis. Preventive medicine,56(5), 245-253.

doi:10.1016/j.ypmed.2013.01.013

Purdie, N., Hattie, J., \& Douglas, G. (1996). Student conceptions of learning and their use of self-regulated learning strategies: A cross-cultural comparison. Journal of Educational Psychology, 88, 87-100. doi:10.1037/0022-0663.88.1.87

Rosenthal, R. (1990). How are we doing in soft psychology? American Psychologist, 45, 775 776. doi:10.1037/0003-066X.45.6.775

Sallis, J. F., Buono, M. J., Roby, J. J., Micale, F. G., \& Nelson, J. A. (1993). Seven-day recall and other physical activity self-reports in children and adolescents. Medicine and Science in Sports and Exercise, 25(1), 99-108.

Sallis, J.F., and Owen, N. (1999). Physical Activity and Behavioral Medicine. Thousand Oaks, CA: Sage.

Schunk, D. H., \& Swartz, C. W. (1993)a. Goals and progress feedback: Effects on selfefficacy and writing achievement. Contemporary Educational Psychology, 18, 337354. doi:10.1006/ceps.1993.1024

Schunk, D. H., \& Swartz, C. W. (1993)b. Writing strategy instruction with gifted students: Effects of goals and feedback on self-efficacy and skills. Roeper Review, 15, 225-230. doi:10.1080/02783199309553512

Schunk, D. H. (2001). Social cognitive theory and self-regulated learning. In B. J. Zimmerman \& D. H. Schunk (Eds.), Self-regulated learning and academic achievement: Theoretical perspectives (2nd ed., pp. 125-151). Mahwah, NJ: Lawrence Erlbaum Associates, Inc.

Schunk, D. H., \& Ertmer, P. A. (1999). Self-regulatory processes during computer skill acquisition: Goal and self-evaluative influences. Journal of Educational Psychology, 91, 251. doi:10.1037/0022-0663.91.2.251 
SELF-REGULATED LEARNING \& ADOLESCENT PHYSICAL ACTIVITY

Steenkamp, J. B. E., \& Baumgartner, H. (2000). On the use of structural equation models for marketing modeling. International Journal of Research in Marketing, 17(2), 195-202.

Steiger, J. H. (1990). Structural model evaluation and modification: An interval estimation approach. Multivariate Behavioral Research, 25, 173-180. doi:10.1207/s15327906mbr2502_4

Stevenson, H. W., \& Lee, S. Y. (1996). The academic achievement of Chinese students. In Bond, Michael Harris (Ed), (1996). The handbook of Chinese psychology, (pp. 124142). New York, NY, US: Oxford University Press.

Tangney, J. P., Baumeister, R. F., \& Boone, A. L. (2004). High self-control predicts good adjustment, less pathology, better grades, and interpersonal success. Journal of personality, 72(2), 271-324.

Telama, R., Yang, X., Viikari, J., Välimäki, I., Wanne, O., \& Raitakari, O. (2005). PA from childhood to adulthood: a 21-year tracking study. American Journal of Preventative Medicine, 28, 267-73. doi:10.1016/j.amepre.2004.12.003

Toering, T.T, Elferink-Gemser, M.T., Jordet, G., \& Visscher, C. (2009). Self-regulation and performance level of elite and non-elite youth soccer players. Journal of Sport Sciences, 27, 1509-1517. doi: 10..1080/02640410903369919.

Toering, T., Elferink-Gemser, M. T., Jonker, L., van Heuvelen, M. J., \& Visscher, C. (2012). Measuring self-regulation in a learning context: Reliability and validity of the SelfRegulation of Learning Self-Report Scale (SRL-SRS). International Journal of Sport and Exercise Psychology, 10, 24-38. doi:10.1080/1612197X.2012.645132

Triandis, H. C. (1995). Individualism \& collectivism. Westview press.

Tudor-Locke, C., Ainsworth, B. E., \& Popkin, B. M. (2001). Active commuting to school. Sports Medicine, 31, 309-313. doi: 0112-1642/01/0005/\$22.00/0 
SELF-REGULATED LEARNING \& ADOLESCENT PHYSICAL ACTIVITY

Turingan, J. P., \& Yang, Y. C. (2009). A cross-cultural comparison of self-regulated learning skills between Korean and Filipino college students. Asian Social Science, 5, 3. doi: $10.5539 / \operatorname{ass} . v 5 \mathrm{n} 12 \mathrm{p} 3$

Yu, C. C. W., Chan, S., Cheng, F., Sung, R. Y. T., \& Hau, K. T. (2006). Are physical activity and academic performance compatible? Academic achievement, conduct, physical activity and self-esteem of Hong Kong Chinese primary school children. Educational Studies, 32(4), 331-341. doi:10.1080/03055690600850016

Warburton, D. E., Nicol, C. W., \& Bredin, S. S. (2006). Health benefits of physical activity: the evidence. Canadian Medical Association Journal, 174, 801-809. doi: 10.1503/cmaj.051351

Winters, E.R., Petosa, R.L., \& Charlton,T.E. (2003). Using social cognitive theory to explain discretionary 'leisure-time' physical exercise among high school students. Journal of Adolescent Health, 32, 436-442. doi:10.1016/S1054-139X(03)00046-6

World Health Organisation, (2013). Physical Activity. Retrieved March 2013, from http://www.who.int/topics/physical_activity/en/.

Zimmerman, B. (1986). Becoming a Self-Regulated Learner: Which Are the Key Subprocesses? Contemporary Educational Psychology, 11, 307-313. doi:10.1016/0361-476X(86)90027-5

Zimmerman, B. J. (2002) Becoming a Self-Regulated Learner: An Overview. Theory into Practice, 41, 64-70. doi:10.1207/s15430421tip4102_2

Zimmerman, B. J., Moylan, A., Hudesman, J., White, N., \& Flugman, B. (2011). Enhancing self-reflection and mathematics achievement of at-risk urban technical college students. Psychological Test and Assessment Modeling, 53, 141-160. 


\section{Table 1}

Mean, standard deviations and Pearson correlations for moderate, vigorous and moderateto-vigorous intensity PA and self-regulation variables in the $H K(n=475)$ and Scottish samples $(n=393)$

\begin{tabular}{|c|c|c|c|c|c|c|c|c|c|}
\hline \multicolumn{10}{|l|}{ HK } \\
\hline & $\mathrm{N}$ & Mean & SD & Planning & $\begin{array}{l}\text { Self- } \\
\text { monitoring }\end{array}$ & Effort & $\begin{array}{l}\text { Self- } \\
\text { efficacy }\end{array}$ & $\begin{array}{l}\text { Self- } \\
\text { evaluation }\end{array}$ & Reflection \\
\hline $\begin{array}{l}\text { Moderate } \\
\text { PA }\end{array}$ & 475 & 10.93 & 10.88 & $.16^{* *}$ & $.13^{* *}$ & $.14^{* * *}$ & $.15^{* *}$ & $.14^{* * *}$ & $.16^{* *}$ \\
\hline $\begin{array}{l}\text { Vigorous } \\
\text { PA }\end{array}$ & 475 & 20.22 & 16.45 & $.12^{* *}$ & .05 & $.15^{* *}$ & $.18^{* *}$ & $.16^{* *}$ & $.14^{* *}$ \\
\hline Total PA & 475 & 31.14 & 22.26 & $.17^{* *}$ & $.10^{*}$ & $.18^{* *}$ & $.21^{* *}$ & $.19^{* *}$ & $.18^{* *}$ \\
\hline Scotland & & & & & & & & & \\
\hline $\begin{array}{l}\text { Moderate } \\
\text { PA }\end{array}$ & 393 & 17.54 & 12.36 & .02 & .02 & .05 & .091 & .069 & $.128^{*}$ \\
\hline $\begin{array}{l}\text { Vigorous } \\
\text { PA }\end{array}$ & 393 & 31.04 & 20.04 & $.14^{* *}$ & .08 & $.19^{* *}$ & $.23^{* *}$ & .08 & $.19^{* * *}$ \\
\hline Total PA & 393 & 48.58 & 26.01 & $.12^{*}$ & .08 & $.17^{* *}$ & $.22^{* *}$ & .09 & $.21^{* *}$ \\
\hline
\end{tabular}


Table 2

Means and standard deviations for self-regulatory components and total SRL score

\begin{tabular}{llllllll}
\hline & Planning & $\begin{array}{l}\text { Self- } \\
\text { monitoring }\end{array}$ & Effort & $\begin{array}{l}\text { Self- } \\
\text { efficacy }\end{array}$ & $\begin{array}{l}\text { Self- } \\
\text { evaluation }\end{array}$ & Reflection & Total SRL score \\
\hline Scotland & $2.51(.50)$ & $2.61(.56)$ & $2.77(.55)$ & $2.67(.50)$ & $3.51(.73)$ & $3.80(.59)$ & $132.93(21.41)$ \\
HK & $2.52(.52)$ & $2.45(.60)$ & $2.71(.55)$ & $2.60(.56)$ & $3.32(.64)$ & $3.65(.63)$ & $86.93(12.49)$ \\
$\begin{array}{l}\text { Significance } \\
\text { level }(2-\end{array}$ & .931 & $.000^{* *}$ & .116 & .056 & $.000^{* *}$ & $.001^{* *}$ & $.000^{* *}$ \\
tailed) & & & & & & & \\
\hline
\end{tabular}

16

17

18

19

20

21

22

23

24

25

26

27

28

29

30

31

32

33

34

35

36

37

38

39

40

41

42

43

44

45

46

47

48

49

50

51

52

53

54

55

56

57

58

59

60

URL: http://mc.manuscriptcentral.com/rijs 
1

2

3

4

5

6

7

8

9

10

11

12

13

14

15

16

17

18

19

20

21

22

23

24

25

26

27

28

29

30

31

32

33

34

35

36

37

38

39

40

41

42

43

44

45

46

47

48

49

50

51

52

53

54

55

56

57

58

59

60

Table 3

Fit indices for the measurement invariance analysis $(n=899)$

\begin{tabular}{lcccccc}
\hline Model & CMIN/DF & TLI & CFI & ACFI & RMSEA & $\begin{array}{l}\text { RMSEA } \\
(95 \% \text { CI })\end{array}$ \\
\hline $\begin{array}{l}\text { Configural invariance } \\
\text { across groups }\end{array}$ & 8.264 & .88 & .95 & & .09 & {$[.08, .10]$} \\
$\begin{array}{l}\text { Metric invariance across } \\
\text { groups }\end{array}$ & 6.957 & .90 & .95 & .001 & .08 & {$[.07,09]$} \\
$\begin{array}{l}\text { Partial Scalar invariance } \\
\text { across groups }\end{array}$ & 6.957 & .90 & .94 & .006 & .08 & {$[.07, .09]$} \\
$\begin{array}{l}\text { Partial Error invariance } \\
\text { across groups }\end{array}$ & 6.492 & .91 & .94 & .004 & .08 & {$[.07, .09]$}
\end{tabular}

Note: $\mathrm{CMIN} / \mathrm{DF}=$ chi-square divided by its degrees of freedom; TLI = Tucker Lewis Index; CFI = comparative fit index;

$\Delta \mathrm{CFI}=$ change in $\mathrm{CFI} ; \mathrm{RMSEA}=$ root mean square error of approximation; $95 \% \mathrm{CI}=95 \%$ confidence interval. 
Table 4

HMR for HK $(n=473)$ and Scottish $(n=389)$ adolescent moderate-to-vigorous intensity PA

\begin{tabular}{|c|c|c|c|c|c|c|c|c|c|c|c|c|}
\hline & HK & & & & & & Scotlan & & & & & \\
\hline Model & $\mathrm{B}$ & $\mathrm{SE}$ & Beta & $t$ & $R^{2}$ & $\Delta R^{2}$ & $\mathrm{~B}$ & $\mathrm{SE}$ & Beta & $t$ & $R^{2}$ & $\Delta R^{2}$ \\
\hline 1 & & & & & .040 & $.04 * *$ & & & & & .08 & $.08 * *$ \\
\hline Constant & 60.32 & 9.03 & & 6.68 & & & 118.78 & 12.74 & & $9.32 * *$ & & \\
\hline Gender & -7.91 & 2.06 & -.174 & $-3.84 * *$ & & & -8.21 & 2.56 & -.16 & $-3.20 * *$ & & \\
\hline Age & -1.22 & .57 & -.097 & $-2.15 * *$ & & & -4.17 & .90 & -.23 & $-4.63 * *$ & & \\
\hline 2 & & & & & .07 & $.03 * *$ & & & & & .13 & $.05^{* *}$ \\
\hline Constant & 37.20 & 10.56 & & 3.52 & & & 88.83 & 14.11 & & $6.29 * *$ & & \\
\hline Gender & -6.95 & 2.04 & -.153 & $-3.40 * *$ & & & -7.31 & 2.51 & -.14 & $-2.91 * *$ & & \\
\hline Age & -1.05 & .56 & -.083 & -1.86 & & & -4.20 & .88 & -.23 & $-4.78 * *$ & & \\
\hline Self-efficacy & 7.33 & 1.81 & .183 & $4.06 * *$ & & & 10.85 & 2.41 & .22 & $4.49 * *$ & & \\
\hline 3 & & & & & .07 & .00 & & & & & .13 & .00 \\
\hline Constant & 35.77 & 10.77 & & 3.32 & & & 90.58 & 14.25 & & $6.36 * *$ & & \\
\hline Gender & -6.92 & 2.04 & -.152 & $-3.39 * *$ & & & -7.11 & 2.52 & -.14 & $-2.82 * *$ & & \\
\hline Age & -1.06 & .56 & -.084 & -1.8 & & & -4.22 & .88 & -.23 & $-4.79 * *$ & & \\
\hline Self-efficacy & 6.17 & 2.47 & .154 & $2.50 * *$ & & & 12.96 & 3.41 & .26 & $3.80 * *$ & & \\
\hline Planning & 1.83 & 2.66 & .042 & .69 & & & -2.95 & 3.36 & -.06 & -.88 & & \\
\hline 4 & & & & & .08 & .00 & & & & & .13 & .00 \\
\hline Constant & 32.32 & 11.00 & & 2.94 & & & 90.04 & 14.56 & & $6.19 * *$ & & \\
\hline Gender & -6.87 & 2.05 & -.151 & $-3.35 * *$ & & & -7.14 & 2.53 & -.14 & $-2.82 * *$ & & \\
\hline Age & -1.04 & .57 & -.083 & -1.82 & & & -4.21 & .89 & -.23 & $-4.71 * *$ & & \\
\hline Self-efficacy & 4.92 & 2.61 & .123 & 1.88 & & & 12.57 & 3.98 & .25 & $3.16 * *$ & & \\
\hline Planning & 1.11 & 3.23 & .026 & .34 & & & -3.23 & 4.02 & -.07 & -.80 & & \\
\hline Effort & 3.69 & 2.37 & .091 & 1.56 & & & .61 & 3.45 & .01 & .18 & & \\
\hline Self-monitoring & -.78 & 2.39 & -.021 & -.37 & & & .16 & 3.23 & .00 & .05 & & \\
\hline 5 & & & & & .09 & $.02 * *$ & & & & & .15 & $.02 * *$ \\
\hline Constant & 19.18 & 11.66 & & 1.65 & & & 70.93 & 15.81 & & 4.49 & & \\
\hline Gender & -7.18 & 2.03 & -.158 & $-3.53 * *$ & & & -7.34 & 2.51 & -.14 & $-2.92 * *$ & & \\
\hline Age & -1.00 & .56 & -.080 & -1.78 & & & -4.01 & .89 & -.22 & $-4.51 * *$ & & \\
\hline Self-efficacy & 4.45 & 2.59 & .111 & 1.71 & & & 11.98 & 3.97 & .24 & $3.02 * *$ & & \\
\hline Planning & -1.24 & 3.36 & -.029 & -.37 & & & -3.23 & 4.05 & -.07 & -.80 & & \\
\hline Effort & 2.16 & 2.39 & .053 & .90 & & & -.42 & 3.47 & -.01 & -.12 & & \\
\hline Self-monitoring & -2.32 & 2.45 & -.062 & -.95 & & & .39 & 3.31 & .01 & .12 & & \\
\hline Self-evaluation & 4.21 & 2.24 & .119 & 1.88 & & & -1.65 & 2.34 & -.05 & -.70 & & \\
\hline Reflection & 3.87 & 1.73 & .109 & $2.24 * *$ & & & 6.93 & 2.33 & .16 & $2.98 * *$ & & \\
\hline
\end{tabular}

Note: B, unstandardised coefficients; SE, standard error; Beta, standardised coefficients; ${ }^{* *} p<.01$ 


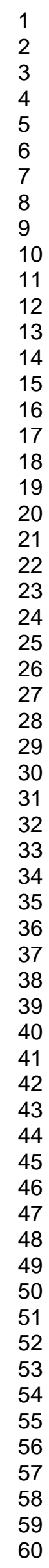

37 